\title{
PREVALENCE OF SEXUAL DYSFUNCTION AND SEXUAL ATTITUDES AMONG POSTMENOPAUSAL FEMALE PATIENTS IN PHRAMONGKUTKLAO HOSPITAL
}

\author{
Kritsada Srithanaphakarangkul
}

Department of Obstetrics \& Gynecology, Phramongkutklao Hospital, Bangkok, Thailand

\begin{abstract}
Background: Sexual function and attitudes towards sexuality among postmenopausal women have been investigated in many ethnicities. Nonetheless, a limited number of studies have been conducted among Thai postmenopausal women.

Objective: The study aimed to determine the prevalence of sexual dysfunction and sexual attitudes among Thai postmenopausal women.

Methods: A descriptive cross-sectional study was conducted from January $1^{\text {st }}, 2018$ to July $31^{\text {st }}, 2018$ at the Gynecologic Outpatient Department, Phramongkutklao Hospital. In all, 293 postmenopausal women, aged 45 years or over were recruited in the study. The Thai version Female Sexual Function Index (Thai-FSFI) questionnaire was used to evaluate the sexual function for which scores less than 26.5 constituted sexual dysfunction. To assess attitudes, validated questionnaires were used to evaluate using scores 0-8, 9-16 and 17-24 as negative, neutral and positive, respectively.

Results: The participants' mean age was $55.53 \pm 5.07$ years. The prevalence of sexual dysfunction was $96.59 \%$ (282/293), which was significantly higher compared with those with normal sexual function. Postmenopausal women with sexual dysfunction were significantly older $(p<0.001)$, had a longer menopause period $(p=0.001)$, had spouses at old age $(p<0.001)$ and had underlying diseases $(p=0.034)$ significantly more than those with normal sexual function. Regarding sexual attitudes, $52.56 \%$ had positive attitudes while $4.44 \%$ had negative attitudes.

Conclusion: Sexual dysfunction is a common problem among Thai postmenopausal women. However, most had positive sexual attitudes.
\end{abstract}

Keywords : Sexual dysfunction, Attitude, Postmenopausal women, Female sexual function index

J Southeast Asian Med Res 2019; 3(2): 82-87.

http://www.jseamed.org

Correspondence to:

Srithanaphakarangkul K, Department of Obstetrics \& Gynecology, Phramongkutklao Hospital, Bangkok 10400 Thailand

e-mail:kritsada@pcm.ac.th 


\section{Introduction}

Sexuality is defined as feelings, attraction and behaviors between two people. Both mental and physical health are factors which impact positive sexual attitudes. Preparing before having sex includes coaxing and proper timing to make it more enjoyable. Regarding the Masters and Johnson model, ${ }^{(2)}$ the sexual response cycle consists of four phases, i.e., the first stage, excitement or arousal phase, of which the sexual response involves increasing blood flow and nerve stimulation in the pelvic area. Among women, production of vaginal lubrication and sexual tension can occur. The second stage or plateau phase occurs after stimulation in phase 1 has been intensified so that the vagina and clitoris become highly sensitive. The third stage or orgasmic phase is the climax of the sexual response during which the heart rate and blood pressure are at their highest. The fourth stage or resolution phase occurs when the body returns to its normal level of functioning. After this phase, women do not need the refractory period; however, men do.

Based on Kaplan's theory about sexual needs, a woman's sexual response is divided in three parts consisting of desire, arousal and orgasm. ${ }^{(3)}$ Sexual activity can occur at any age but mostly occurs during reproductive age. Having a longer life leads not only to a rise in number of aging population but also increased sexual problems.

Sexual problems among the elderly result from many factors. Among females, the major factor is physiological change due to menopause. Due to decreasing sex hormones, menopausal women suffer from vaginal dryness, vasomotor symptoms, mood changes and increase of underlying diseases such as heart disease, hypertension and osteoporosis. In addition, some cultures believe that the elderly shouldn't be interested in sexual activity. For these reasons, in some countries, most elderly have little interest in sex or wouldn't want to have sex.

Related studies showed that $84.1 \%$ of Paraguayan middle age females were sexually active while $25.6 \%$ experienced sexual dysfunction. ${ }^{(4)}$ On the other hand, other studies have reported $81.5 \%$ of elderly women experienced sexual dysfunction and $62.1 \%$ had negative sexual attitudes. ${ }^{(5)}$ Studies have confirmed that sexual desire decreased among postmenopausal women; ${ }^{(6,8)}$ however, sexual dysfunction has been increasing especially among women having underlying diseases. ${ }^{(7)}$ Sexual dysfunction is a common problem among postmenopausal women decreasing the quality of life. ${ }^{(9)}$ However, the prevalence of sexual dysfunction differs among different countries and study areas.

Due to limitations of data among Thai postmenopausal women, the main purpose of this study was to evaluate the prevalence of sexual dysfunction and attitudes among postmenopausal women attending the Gynecologic Outpatient Department at Phramongkutklao Hospital.

\section{Methods}

This cross-sectional study was approved by the Institutional Review Board, Royal Thai Army Medical Department. Postmenopausal women attending the Outpatient Department of Gynecology, Phramongkutklao Hospital from April $1^{\text {st }}$, 2018 to July $31^{\text {st }}, 2018$ were recruited in the study. Inclusion criteria comprised Thai women with naturally occurring menopause and those with surgical menopause who were at least 45 year old and had sexual activity more than once monthly. Exclusion criteria included those unable to read and write Thai, presenting physically defect or having psychological problems.

A total of 293 participants were recruited in the study. After the complete informed consent was processed, the participants were requested to complete two parts of self-questionnaires of the Thai version Female Sexual Function Index (Thai-FSFI). The first part covered female sexual function index (FSFI) using a validated questionnaire originally created by Rosen et al. ${ }^{(10)}$ This questionnaire was translated to the Thai version or Thai-FSFI. ${ }^{(11)}$ The Thai-FSFI was divided in six sections consisting of desire, arousal, lubrication, orgasm, sexual satisfaction and pain. ${ }^{(12)}$ Of 19 questions, scores totaled 36 . The minimal score is 1.2 and the maximum score is 6 in each section. Scores less than 26.5 constitute sexual dysfunction. The second part comprised the sexual attitude questionnaire consisting of 12 questions; each question is scored 0 to 2 . Thus, the minimum score is 0 and the maximum score is 24 . Scores of 0 to 8 are considered negative attitudes, scores 9 to 16 are neutral and scores over 16 constitute positive attitudes.

All data were analyzed using SPSS, Version 17.0. 
Descriptive statistics were used to analyze participant's characteristics and attitudes. The Chi-square test and Fisher's exact test were used to compare frequency distribution of categorical variables. All tests found significance at $p<0.05$.

\section{Results}

The prevalence of sexual dysfunction among postmenopausal female patients was $96.5 \%$. The demographic and baseline disease characteristics of the postmenopausal female patients are shown in Table 1. The mean participants' age was $55.32 \pm 5.13$ years and the mean time of menopausal period was $5.49 \pm 3.8$ years. According to the results, approximately one third of participants had underlying diseases $(32.76 \%)$, experienced sexual abuse $(1.37 \%)$ and received hormonal therapy (23.89\%). The participants' spouse mean age was $56.85 \pm 5.98$ years.
As shown in Table 2, 89.42\% had no underlying diseases, $16.04 \%$ consumed alcohol and $7.17 \%$ smoked. As shown in Tables 3 and 4, age of postmenopausal women and their spouse in the sexual dysfunction group was significantly higher than those of the normal sexual function group $(p<0.001)$. A significant difference of mean menopausal period was found between the normal sexual function group (2.1 \pm 1.66 years) and the sexual dysfunction group $(5.61 \pm 3.80$ years $)(p=0.001)$. In addition, subjects with sexual dysfunction presenting underlying diseases significantly differed compared with the normal sexual function group ( $p=0.034)$.

Regarding sexual attitudes, approximately one half of the participants (52.56\%) had positive attitudes, $43 \%$ had neutral attitudes, but only $4.44 \%$ had negative attitudes (data not shown).

Table 1. Characteristics of the enrolled participants $(\mathrm{N}=293)$

\begin{tabular}{lcc}
\hline \multicolumn{1}{c}{ Characteristics } & Number & $\%$ \\
\hline $\begin{array}{l}\text { Age ( years) } \\
\text { mean } \pm \text { SD }\end{array}$ & $55.53 \pm 5.07$ \\
$\begin{array}{l}\text { Menopausal period (years) } \\
\text { mean } \pm \text { SD }\end{array}$ & 148 & $5.49 \pm 3.80$ \\
Education & 163 & 50.51 \\
Working & 197 & 55.63 \\
Underlying diseases & 3 & 67.24 \\
Alcohol drinking & 3 & 1.02 \\
Smoking & 3 & 1.02 \\
Sexual abuse & & 23.89 \\
Hormonal used & 70 & 1.37 \\
\hline
\end{tabular}


Table 2. Characteristics of spouses $(\mathrm{N}=293)$

\begin{tabular}{lcc}
\hline Characteristics & Number & $\%$ \\
\hline Age (years) & & $56.86 \pm 5.98$ \\
mean \pm SD & 31 & 10.58 \\
Underlying diseases & 47 & 16.04 \\
Alcohol drinking & 21 & 7.17 \\
Smoking & & \\
\hline
\end{tabular}

Table 3. Characteristics of postmenstrual women associated with sexual dysfunction

\begin{tabular}{|c|c|c|c|}
\hline Characteristics & $\begin{array}{l}\text { Normal } \\
11(\%)\end{array}$ & $\begin{array}{c}\text { Sexual Dysfunction } \\
282(\%)\end{array}$ & $p$-value \\
\hline $\begin{array}{l}\text { Age (years) } \\
\text { mean } \pm \mathrm{SD}\end{array}$ & $49.30 \pm 2.67$ & $55.53 \pm 5.07$ & $<0.001$ \\
\hline $\begin{array}{l}\text { Menopausal period, years } \\
\text { mean } \pm \text { SD }\end{array}$ & $2.10 \pm 1.66$ & $5.61 \pm 3.80$ & 0.001 \\
\hline $\begin{array}{l}\text { Education } \\
\text { Yes } \\
\text { No }\end{array}$ & $\begin{array}{l}6(4.05) \\
5(3.45)\end{array}$ & $\begin{array}{l}142(95.95) \\
140(96.55)\end{array}$ & 0.750 \\
\hline $\begin{array}{l}\text { Working } \\
\text { Yes } \\
\text { No }\end{array}$ & $\begin{array}{l}8(4.91) \\
3(2.31)\end{array}$ & $\begin{array}{l}155(95.09) \\
127(97.69)\end{array}$ & 0.194 \\
\hline $\begin{array}{l}\text { Underlying diseases } \\
\text { Yes } \\
\text { No }\end{array}$ & $\begin{array}{c}10(5.08) \\
1(0.04)\end{array}$ & $\begin{array}{l}187(94.92) \\
95(99.06)\end{array}$ & 0.034 \\
\hline $\begin{array}{l}\text { Drinking alcohol } \\
\text { Yes } \\
\text { No }\end{array}$ & $\begin{array}{c}0(0) \\
11(1.02)\end{array}$ & $\begin{array}{c}3(100) \\
279(98.98)\end{array}$ & 1.000 \\
\hline $\begin{array}{l}\text { Smoking } \\
\text { Yes } \\
\text { No }\end{array}$ & $\begin{array}{c}0(0) \\
11(1.02)\end{array}$ & $\begin{array}{c}3(100) \\
279(98.98)\end{array}$ & 1.000 \\
\hline $\begin{array}{l}\text { Sexual abuse } \\
\text { Yes } \\
\text { No }\end{array}$ & $11(3.81)$ & $\begin{array}{c}4(100) \\
278(96.19)\end{array}$ & 1.000 \\
\hline $\begin{array}{l}\text { Hormonal used } \\
\text { Yes } \\
\text { No }\end{array}$ & $\begin{array}{l}3(4.29) \\
8(3.59)\end{array}$ & $\begin{array}{c}67(95.71) \\
215(96.41)\end{array}$ & 0.707 \\
\hline
\end{tabular}


Table 4. Characteristics of participant' spouse associated with sexual dysfunction (N=293)

\begin{tabular}{lccc}
\hline Characteristics & $\begin{array}{c}\text { Normal } \\
\mathrm{N}=11(\%)\end{array}$ & $\begin{array}{c}\text { Sexual } \\
\text { Dysfunction } \\
\mathrm{N}=282(\%)\end{array}$ & $p$-value \\
\hline Age years (mean $\pm \mathrm{SD})$ & $49.70 \pm 3.86$ & $57.11 \pm 5.98$ & $<0.001$ \\
Underlying diseases & $0(0)$ & $31(10.99)$ & 1.000 \\
Alcohol drinking & $0(0)$ & $47(16.67)$ & 1.000 \\
Smoking & $0(0)$ & $21(7.45)$ & 1.000 \\
\hline
\end{tabular}

\section{Discussion}

When compared with related studies, ${ }^{(4)}$ similar results were observed for which sexual dysfunction was a common problem among postmenopausal women. In this study, the prevalence of sexual dysfunction among postmenopausal women attending the Outpatient Department of Gynecology, Phramongkutklao Hospital was rather high (96.59\%). The prevalence was much higher when compared with studies in other countries; however, approximately one half still had positive sexual attitudes. This could have resulted from ethnicity, population characteristics, religion, cultural differences, social problem and using different questionnaires to evaluate sexual dysfunction. Those reporting positive attitudes could have recognized sexuality as a natural part of life.

Participant's and spouse's age were important factors concerning sexual dysfunction. Among postmenstrual women, decreasing estrogen levels could have affected sexual function. Experiencing vasomotor symptoms and vaginal dryness would lead to decreased sex drive or having unpleasurable sex due to dyspareunia. The longer the postmenopausal period they had, the higher the prevalence of sexual dysfunction was found. Moreover, not only decreased hormones and sexual emotion, but also culture may affect sexual activity and function especially among Thai postmenopausal women. Another important factor affecting sexual function was underlying diseases for which a higher prevalence of sexual function was observed among postmenopausal women. Some diseases could have made them too tired or stressed including certain prescription drugs which would have decreased the sex drive and could interfere with sexual activity. To handle the problem, providing sexual knowledge could decrease sexual dysfunction among females and their spouses.

The strength of this study was the use of a validated standard questionnaire, used worldwide to evaluate sexual function. Because data of the prevalence of sexual dysfunction among Thai postmenopausal women are limited, the preliminary data in this study provided greater understanding of importance issues concerning mental and physical health status of Thai postmenopausal women aged 45 years or over. Nevertheless, some limitations were observed. The results from this study could only represent one hospital-based population. A high prevalence of sexual dysfunction could have resulted from the selection of participants. This study evaluated menopausal status among both natural and surgical postmenopausal Thai women at the Gynecologic Outpatient Department sharing common health problems. Other information of spouses' health status could not be obtained because questionnaires were not assessed by the spouses themselves. Moreover, precise details about sexual attitudes were not assessed; thus, reasons for having positive, neutral or negative sexual attitudes in the study group remained unclarified. Further information from a qualitative study of their attitudes, sexual knowledge and behaviors would be useful to reduce or prevent sexual dysfunction. 
In conclusion, sexual dysfunction was observed to be a common problem with high prevalence among postmenopausal female patients in Phramongkutklao Hospital. Postmenopausal women with sexual dysfunction were significantly older, had a longer menopause period, had elderly spouses and had more underlying diseases than those who experienced normal sexual function. However, about one half had positive sexual attitudes.

\section{Acknowledgement}

The study was supported by the Department of Obstetrics and Gynecology, Phramongkutklao Hospital, Bangkok, Thailand.

\section{References}

1. Anek AP. Sexuality In Anek AP. the editor. Human sexuality in Obstetrics and Gynecology $1^{\text {st }}$. Bangkok: Pimpdee; 2005.1-9

2. Master EH, Johnson VE. Human sexual response. Boston: Little Brown; 1966.

3. Kaplan HS. The new sex therapy. London: Bailliere Trindall, 1974.

4. Sanchez SC, Cherdraui P, Perez-Lopez FR, Ortiz-Benegas ME, Palacios-De Franco Y. Evaluation of sexuality in Paraguayan mid-aged female urban population using the six-item Female Sexual Function Index. Climacteri . 2016; 19: 256-60

5. Jamali S, Ratimanian A, Javadpoor S. Examining the sexual function and related attitudes among aged

5. women: A cross-sectional study. Int J Reprod Biomed (Yazd) 2016; 14: 29-38.

Woods NF, Mitchell ES, Smith-Di Julio K. Sexual desire during the menopausal transition and early post-

6. menopause: observations from the Seattle Midlife Women's Health Study. J Womens Health (Larchmt) 2010; 19: 209-18.

Silva GM, Lima SM, Moraes JC. Evaluation of sexual function in postmenopause women with metabolic

7. syndrome. Rev Bras Ginecol Obstet 2013; 35: 301-8. Dhillon HK, Singh HJ, Ghaffar NA. Sexual function in menopausal women in Kelantan, Malaysia. Maturitas

8. $2005 ; 52: 256-63$.

Nazarpour S, Simbar M, Ramezani Tehrani F, AlaviMajd H. Quality of life and sexual function in post-

9. menopausal women. J Women Aging 2017: 1-11.

Rosen R, Brown C, Heiman J, Leiblum S, Meston C, Shabsigh R, et al. The Female Sexual Function Index

10. (FSFI): A multidimensional self-report instrument for the assessment of female sexual function. J Sex Marital Ther 2000; 26: 191-208.

Chayachinda C, Titapant V, Ungkanungdecha A. Dyspareuria and sexual dysfunction after vaginal delivery

11. in Thai primiparous women with episiotomy. J Sex Med 2015; 12: 1275-82.

Kerdarunsuksri A, Manusirivithaya S. Attitudes and sexual function in Thai pregnant women. J Med Assoc

12. Thai 2010; $93: 265-71$. 